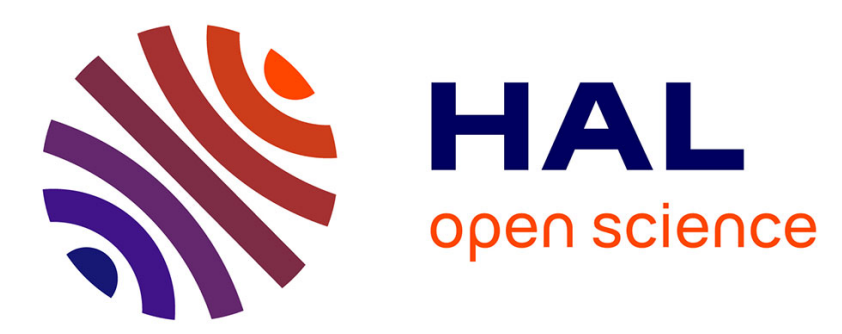

\title{
An optimization-based control approach for reliable microgrid energy management under uncertainties
}

\author{
Ionela Prodan, Enrico Zio
}

\section{To cite this version:}

Ionela Prodan, Enrico Zio. An optimization-based control approach for reliable microgrid energy management under uncertainties. ISEPS 2013, Nov 2013, Bucharest, Romania. pp.1-4. hal-00912004

\section{HAL Id: hal-00912004 \\ https://hal-centralesupelec.archives-ouvertes.fr/hal-00912004}

Submitted on 1 Dec 2013

HAL is a multi-disciplinary open access archive for the deposit and dissemination of scientific research documents, whether they are published or not. The documents may come from teaching and research institutions in France or abroad, or from public or private research centers.
L'archive ouverte pluridisciplinaire HAL, est destinée au dépôt et à la diffusion de documents scientifiques de niveau recherche, publiés ou non, émanant des établissements d'enseignement et de recherche français ou étrangers, des laboratoires publics ou privés. 


\title{
An optimization-based control approach for reliable microgrid energy management under uncertainties
}

\author{
Ionela Prodan ${ }^{1}$, Enrico Zio ${ }^{1,2}$
}

\begin{abstract}
This paper proposes an optimizationbased control approach for microgrid energy management. For exemplification of the approach consider a microgrid system connected to an external grid via a transformer and containing a local consumer, a renewable generator (wind turbine) and a storage facility (battery). The objective of minimizing the costs is achieved through a predictive control framework for scheduling the battery usage in the microgrid system. The proposed control framework takes into account cost values, power consumption, generation profiles as well as functional constraints under uncertainties in the wind speed profile. Simulation results using real numerical data are presented for a reliability test system.
\end{abstract}

\section{INTRODUCTION}

Future intelligent electricity grids attract increasing public attention during the last years. Green (solar and wind) energy production is supposed to increase significantly in the coming years. This requires a "smartgrid" approach in order to deal with distributed production/intermittent variations of output and optimal scheduling of demand [1].

In the present paper, we introduce an original optimization-based control approach to efficiently manage the energy production in a microgrid system.

Various approaches for the energy management within a microgrid are reported in the literature. For example, [2], [3] propose an agent-based modeling approach to model microgrids and to analyze by simulation the interactions between individual intelligent decision-makers. Energy management of hybrid renewable energy generation was proposed in [4], [5]. In these papers, the long-term goals are focused on the efficient use of electricity within microgrids, e.g., the planning of battery scheduling to store the electricity locally generated from renewable sources and reuse it during periods of high electricity demand. It is important to mention that in these works the decision framework was developed under deterministic conditions, e.g., those of a typical day in summer, and without taking into account explicitly the dynamics of the individual components of the microgrids.

The starting point of our work is that a widely used technique in the control community to manage the dynamics of systems affected by uncertainties in

1 Chair on Systems Science and the Energetic Challenge, European Foundation for New Energy - EDF, Ecole Centrale Paris, Grande Voie des Vignes 92295 and Supélec, Gif sur Yvette 91192, France. 2 Department of Energy, Politecnico di Milano, Milano, Italy. \{ionela.prodan,enrico.zio\}@ecp.fr. the behavior of their components is Model Predictive Control (MPC) (see, for instance, [6] for basic notions about MPC), due to its ability to handle control and state constraints while offering good performance specifications. Typically, in MPC, the objective (or cost) function penalizes deviations of the states and inputs from their reference values, while the constraints are enforced explicitly. Due to its versatility, MPC has had a successful record in industrial applications, to mention just a few recent ones: for refrigeration systems [7], for power production plants [8] and transportation networks [9].

In the present paper, we address the problem of reliable energy management of a microgrid by proposing a predictive control framework. The microgrid considered as example is inspired from [3] and is connected to an external grid via a transformer and contains a local consumer, a renewable generator (wind turbine) and a storage facility (battery). The underlying management setting is one of multi-criteria decision-making for battery scheduling with the objectives of increasing the utilization rate of the battery during high electricity demand (i.e., decrease of the electricity purchase from the external grid) and increasing the utilization rate of the wind turbine for local use (i.e., increase of the consumer independence from the external grid). With respect to previous work [10], [3], we propose a more realistic model of the battery. In particular, we assume the existence of a leakage current and provide a switched model with "charge" and "discharge" functioning modes.

The remainder of the paper is organized as follows. Section II presents the components of the microgrid system and Section III describes the predictive control mechanism used for battery scheduling within the microgrid. Some simulation reasults are presented in Section IV and the conclusions are drawn in Section V

\section{System AND MODEL DESCRIPTION}

Consider a microgrid as in Fig. 1, which includes a local consumer (e.g., large cooling houses), a renewable generator (e.g., wind turbine) and a storage facility (e.g., battery). The microgrid is connected to the external grid via a transformer. The goal is to plan the battery schedule in order to achieve the consumer objectives of increasing the utilization rate of the battery during high electricity demand (i.e., decrease the electricity purchase from the external grid) and the utilization rate 


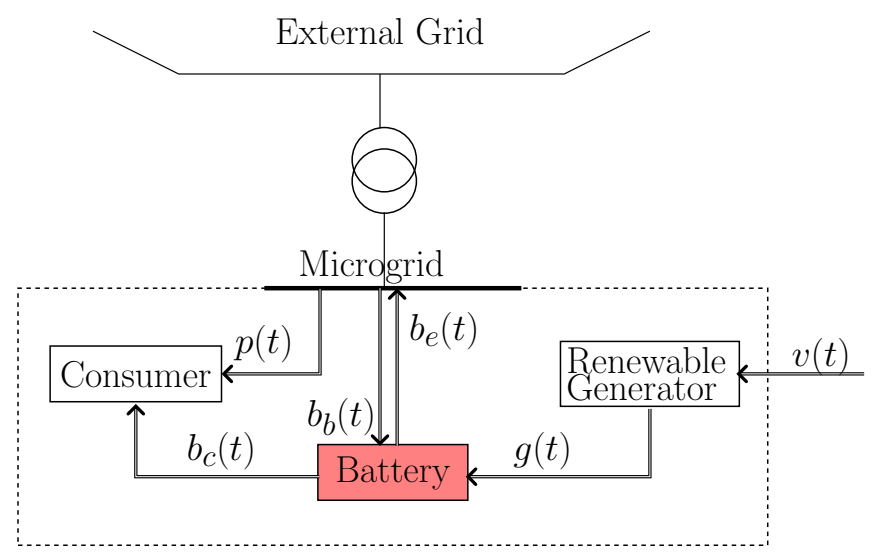

Fig. 1: Microgrid architecture

of the generator for local use (i.e., increase the consumer independence from the external grid).

The interactions between the independent components of the microgrid are one of the most important factors in accomplishing the consumer objectives. More precisely, as shown in Fig. 1 the electricity power transmitted by the renewable generator to the battery at time step $t$ is represented by $g(t) \in \mathbb{R}$, the electricity power transmitted by the battery to the consumer at time step $t$ is represented by $b_{c}(t) \in \mathbb{R}$. In order to maximize the utilization of the battery within the microgrid, the consumer takes electricity from the local renewable generator through the battery. The battery can be charged from the external grid or can also give electricity to the external grid: the electricity power transmitted by the battery to the external grid at time step $t$ is denoted by $b_{e}(t) \in \mathbb{R}$. It is possible to sell electricity to the external grid when the level of charge in the battery is deemed sufficient for covering local needs. The electricity power transmitted by the external grid to the battery at time step $t$ is $b_{b}(t) \in \mathbb{R}$ and the electricity power transmitted by the external grid to the consumer at time $t$ is represented by $p(t) \in \mathbb{R}$. Here, the transformer provides electricity power from the external grid as well as information about the electricity market price, which plays an important role as the consumer can decide to take energy when the price is low. Therefore, the consumer has also the possibility to take electricity from the external grid when the renewable resource is not available (or sufficient).

Finally, the ultimate goal is to control the battery such that all the consumers objectives are fulfilled while considering all the interactions between the independent components of the electrical grid.

\section{A. Dynamic models of the microgrid components}

The controllable component is the battery. For describing its dynamics we consider the following equation:

$$
b(t+1)=b(t)+\left[b_{b}(t)+g(t)-b_{c}(t)-b_{e}(t)\right] \Delta t,
$$

where $b(t) \in \mathbb{R}[W h]$ represents the level of battery charge at time step $t$ and $b_{b}(t) \in \mathbb{R}[\mathrm{W}], g(t) \in \mathbb{R}[\mathrm{W}]$, $b_{c}(t) \in \mathbb{R}[\mathrm{W}], b_{e}(t) \in \mathbb{R}[\mathrm{W}]$ are the electricity power quantities previously described (see also Fig. 1).

Consider also the generator system, whose dynamics can be described as:

$$
g(t+1)=f(g(t), v(t)),
$$

where $f(\cdot, \cdot): \mathbb{R} \rightarrow \mathbb{R}$ is the dynamical function, $g(t) \in \mathbb{R}$ the electricity power given by the generator and $v(t) \in \mathbb{R}$ the wind speed at time step $t$ (here considered of 1 hour). Note that $f(\cdot, \cdot)$ is nonlinear, that is, the output of the generator depends nonlinearly on the wind speed and can be roughly partitioned into three regimes of functioning (starting-up, nominal functioning and dangerous wind levels) as shown in Fig. 2:

$$
g(t)= \begin{cases}0, & \text { if } v<v_{c i}, \\ P_{r} \cdot \frac{v(t)-v_{c i}}{v_{r}-v_{c i}} \cdot \Delta t, & \text { if } v_{c i} \leq v(t)<v_{r}, \\ P_{r} \cdot \Delta t, & \text { if } v_{r} \leq v<v_{c o}, \\ 0, & \text { if } v>v_{c o},\end{cases}
$$

where $v_{c i}[\mathrm{~m} / \mathrm{s}], v_{r}[\mathrm{~m} / \mathrm{s}]$ and $v_{c o}[\mathrm{~m} / \mathrm{s}]$ are the cut-in, rated and cut-off wind speeds, respectively, and $P_{r}[\mathrm{~W}]$ is the rated power of the wind turbine. Uncertainty in the power curve parameters (3) are considered, in the ranges $v_{1} \leq v_{c i} \leq v_{2}, v_{3} \leq v_{r} \leq v_{4}, v_{5} \leq v_{c o} \leq v_{6}$ and $p_{1} \leq P_{r} \leq p_{2}$, where $v_{1}, v_{2}, v_{3}, v_{4}, v_{5}, v_{6}, p_{1}, p_{2} \in \mathbb{R}$.

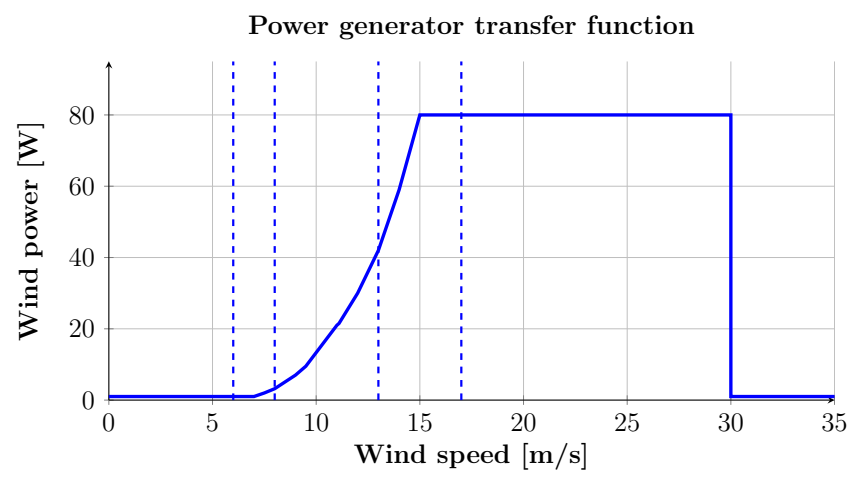

Fig. 2: Reference power curve for the wind turbine as a function of the wind speed.

\section{B. Constraints description}

From Fig. 1 it is seen that the consumer can take electricity from two sources, i.e., the battery and the external grid. Therefore, for a reliable management of the energy system it is necessary to ensure that at time $t$ the electricity purchased from these two sources satisfies the following condition:

$$
b_{c}(t)+p(t) \geq d(t) .
$$

The energy stored in the battery at time $t$ needs to remain between some bounds; consequently, the following 
condition is imposed:

$$
B_{\min } \leq b(t) \leq B_{\max },
$$

where $B_{\min }, B_{\max } \in \mathbb{R}$. The maximum battery capacity, $B_{\max }$, is equal to the rated capacity. The minimum capacity, $B_{\min }$, is determined from the Depth of Discharge (DoD) which is used to describe how deeply the battery is discharged:

$$
B_{\min }=(1-D o D) \times B_{\max } .
$$

Furthermore, the rate of battery charge at time $t$ needs also to remain between some bounds as described by the following condition:

$$
D_{\min } \leq \Delta b(t) \leq D_{\max },
$$

where $D_{\text {min }}, D_{\max } \in \mathbb{R}$.

Finally, constraints on the amount of electricity transmitted to and from the battery as well as to the consumer are imposed:

$$
U_{\min } \leq u(t) \leq U_{\max },
$$

where $U_{\min }, U_{\max } \in \mathbb{R}$ and $u(t)=$ $\left[\begin{array}{llll}p^{T}(t) & b_{e}^{T}(t) & b_{c}^{T}(t) & b_{b}^{T}(t)\end{array}\right]^{T}$ represents the vector of control signals.

\section{Cost function description}

Denote by $e(t) \in \mathbb{R}[\$ / W h]$ the electricity market price at time step $t$. Then, the total energy cost at time $t$ is:

$$
C(t)=e(t) \cdot \Delta t\left[b_{b}(t)+p(t)-b_{e}(t)\right],
$$

where a small value of $C(t)$ means small energy drawn from the external grid.

\section{Profiles of interest}

All the elements of the electrical system are characterized by certain profiles of reference. Arguably, the most important is the profile characterizing the consumer load, denoted as $d(t)$. Taking into account the weekly, daily and hourly variability it is possible to predict a reference load. Therefore, in order to predict the reference load of the consumer we consider a top-down approach based on available statistical measurements of electricity consumption (the real numerical data of a reliability test system task force are found in [11]).

Lastly, we need to provide the evolution of the prices $e(t)$ on the electricity market. Again, we use existing historical data of market prices [11].

\section{OPTIMIZATION-BASED CONTROL MECHANISM FOR BATTERY SCHEDULING}

For a reliable energy management of the microgrid by battery scheduling, we consider the recursive construction of an optimal open-loop control sequence $\mathbf{u}=\left\{u(k), u(k+1), \cdots, u\left(k+N_{\mathrm{p}}-1\right)\right\}$, with $u(k)=$ $\left[\begin{array}{lllll}p^{T}(k) & b_{e}^{T}(k) & b_{c}^{T}(k) & b_{b}^{T}(k)\end{array}\right]^{T}$ over a finite constrained receding horizon, which leads to a feedback control policy by the effective application of the first control action as system input:

$$
u^{*}=\arg \min _{[u(k)]_{t=k: k+N_{p}}} \sum_{t=k}^{k+N_{\mathrm{p}}} C(t) \gamma^{k-t},
$$

subject to the set of constraints:

$$
\begin{aligned}
& b(t+1)=b(t)+\left[b_{b}(t)+g(t)-b_{c}(t)-b_{e}(t)\right] \Delta t, \\
& g(t+1)=f(g(t), v(t)), t=k: k+N_{p}, \\
& b_{c}(t)+p(t) \geq d(t), t=k: k+N_{p}, \\
& B_{\min } \leq b(t) \leq B_{\max }, t=k: k+N_{p}, \\
& D_{\min } \leq \Delta b(t) \leq D_{\max }, t=k: k+N_{p}, \\
& U_{\min } \leq u(t) \leq U_{\max }, t=k: k+N_{p}, \\
& C(t)=e(t) \cdot \Delta t\left[b_{b}(t)+p(t)-b_{e}(t)\right], t=k: k+N_{p},
\end{aligned}
$$

where $\gamma \in(0,1)$ and depending on the values it takes between this interval the cost will decrease more or less fast or not at all.

The strategy used for microgrid energy management is implemented in the following algorithm:

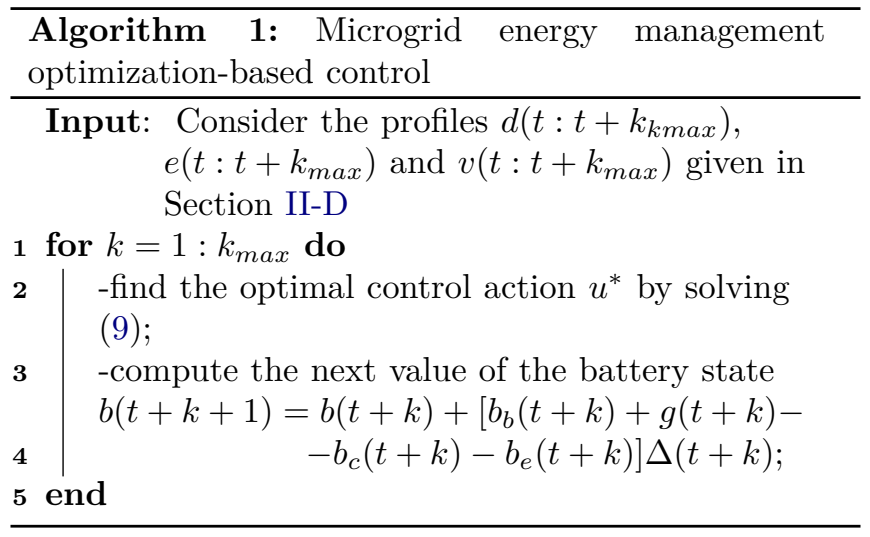

Note that the price $e(t)$ is variable, therefore, in (9) we have a variable cost function. It is important to mention that the increase of the prediction horizon will imply a decrease of the electricity cost taken from the external grid. Also, the increase of the prediction horizon length does not mean that the uncertainty (in the profiles, for example) accumulate. MPC deals very well with this type of problems, where a grater weight can be given to the immediate future costs and a smaller weight to the future ahead cost (i.e., the cost at time $k$ becomes less and less important because the term $\gamma^{k-t}$ is decreasing).

\section{Simulation RESUlts}

We consider the dynamic model (1) for the battery, the discretization step $\Delta t=1$ hour and the profiles described in Section II-D. The battery charge bounds are $B_{\min }=3 \mathrm{kWh}, B_{\max }=6 \mathrm{kWh}$ and the battery charge variation bounds $D_{\min }=-1 \mathrm{kWh}, D_{\max }=1 \mathrm{~kW}$. Also, the control signals $b_{b}(t), b_{c}(t), b_{e}(t), p(t)$ need to stay between some bounds, i.e., $U_{\min }=\left[\begin{array}{llll}0 & 0 & 0 & 0\end{array}\right]^{T} \mathrm{~kW}$, $U_{\max }=\left[\begin{array}{llll}6 & 6 & 6 & 6\end{array}\right]^{T} \mathrm{~kW}$. 
The Figures of this section illustrate several of the signals of interest. First, Fig. 3 shows the level of battery charge.

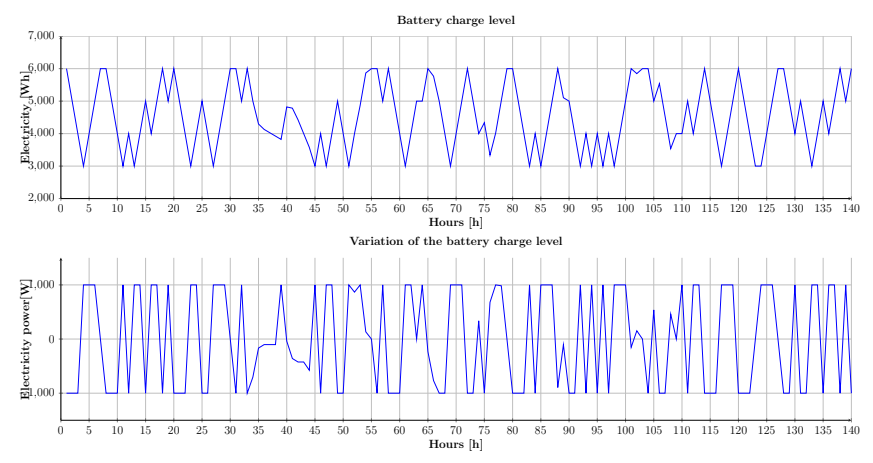

Fig. 3: Battery charge level variation.

Next, Fig. 4 (top right) shows the consumer power demand $d(t)$ (depicted by dotted line mark), power given by the battery to the consumer $b_{c}(t)$ (denoted by plus line mark), the power given by the external grid to the consumer $p(t)$ (depicted in square line mark). Notice that the sum of the last two (i.e., $b_{c}(t)+p(t)$ ) is depicted in dashed line and is always equal to the consumer power demand, which means that the consumer is always receiving the electricity power demanded.

The same Fig. 4 (bottom left) depicts both the power given by the battery to the external grid $b_{e}(t)$ (triangle line mark) and the power given by the external grid to the battery $b_{b}(t)$ (diamond line mark). Also, with minus line mark we plot how much power is drawn overall from the grid $p(t)+b_{b}(t)-b_{e}(t)$ (power given from the external grid to the consumer + power given from the grid to the battery - power given from the battery to the grid).

Finally, Fig. 4 (bottom right) shows the value of the cost at each time instant.
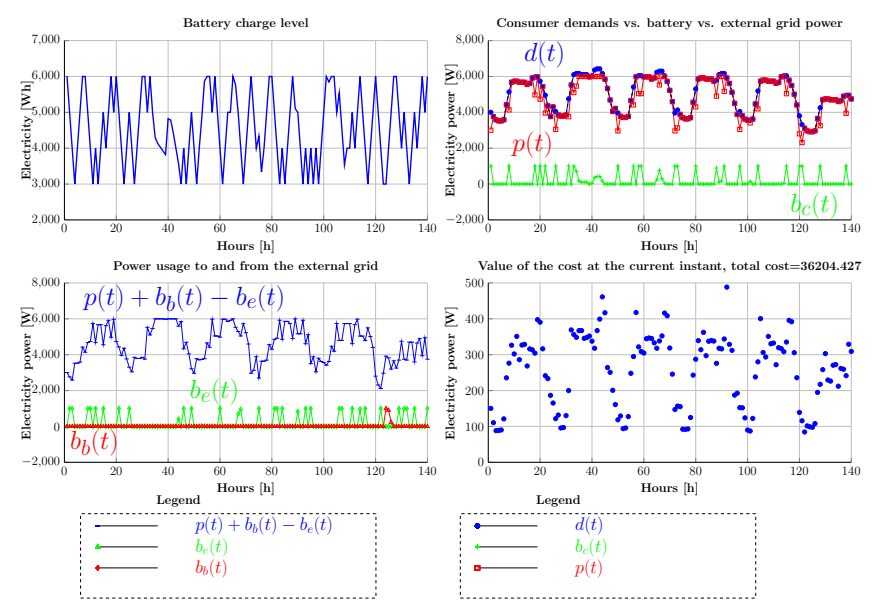

Fig. 4: Power usage.

\section{Conclusions}

In this paper, we propose an approach for reliable microgrid energy management based on receding horizon control to minimize the costs. A scheduling of battery usage is determined. The predictive control framework allows to naturally take into consideration variable cost values, power consumption and generation profiles, as well as functional constraints under uncertainty due to variations in the environment (wind speed). The simulation results using real numerical data for a reliability test system have proved the feasibility of the proposed approach. With the considered management strategy, a good equilibrium between decentralized energy production, energy needs and integration into the grid can be found. Future work will focus on developing a control theory setting to frame the state transition process of degradation/failure/recovery dynamics in microgrid energy systems.

\section{REFERENCES}

[1] K. Alanne and A. Saari, "Distributed energy generation and sustainable development," Renewable and Sustainable Energy Reviews, vol. 10, no. 6, pp. 539-558, 2006.

[2] T. Krause, E. Beck, R. Cherkaoui, A. Germond, G. Andersson, and D. Ernst, "A comparison of nash equilibria analysis and agent-based modelling for power markets," International Journal of Electrical Power \& Energy Systems, vol. 28, no. 9, pp. 599-607, 2006.

[3] E. Kuznetsova, Y. F. Li, C. Ruiz, E. Zio, G. Ault, and l. K. Bel, "Reinforcement learning for microgrid energy management," Energy Journal, 2013, accepted for publication, available upon request.

[4] C. Colson, M. Nehrir, and R. Gunderson, "Multi-agent microgrid power management," in Proceedings of the 18th IFAC World Congress, 2010, pp. 77-82.

[5] Z. Jun, L. Junfeng, W. Jie, and H. Ngan, "A multi-agent solution to energy management in hybrid renewable energy generation system," Renewable Energy, vol. 36, no. 5, pp. 1352-1363, 2011.

[6] J. Rawlings and D. Mayne, "Postface to "model predictive control: Theory and design"," 2011.

[7] T. Hovgaard, L. Larsen, and J. Jorgensen, "Robust economic mpc for a power management scenario with uncertainties," in Proceedings of the 50th IEEE Conference on Decision and Control and European Control Conference (CDC-ECC). IEEE, 2011, pp. 1515-1520.

[8] K. Edlund, J. Bendtsen, and J. Jørgensen, "Hierarchical model-based predictive control of a power plant portfolio," Control Engineering Practice, vol. 19, no. 10, pp. 1126-1136, 2011.

[9] R. Negenborn, B. De Schutter, and J. Hellendoorn, "Multiagent model predictive control for transportation networks: Serial versus parallel schemes," Engineering Applications of Artificial Intelligence, vol. 21, no. 3, pp. 353-366, 2008.

[10] I. Prodan and E. Zio, "Predictive control for a reliable microgrid energy management under uncertainties," Amsterdam, Netherlands, September 29 - October 2, 2013, available upon request.

[11] C. Grigg, P. Wong, P. Albrecht, R. Allan, M. Bhavaraju, R. Billinton, Q. Chen, C. Fong, S. Haddad, and S. Kuruganty, "The ieee reliability test system-1996. a report prepared by the reliability test system task force of the application of probability methods subcommittee," Power Systems, IEEE Transactions on, vol. 14, no. 3, pp. 1010-1020, 1999. 